\title{
SIMULATION AND INTERPRETATION OF MULTIPHASE PROCESSES IN POROUS AND FRACTURED-POROUS MEDIA
}

\author{
R. HELMIG; H. KOBUS and C. BRAUN \\ Institut für Wasserbau \\ University of Stuttgart \\ Pfaffenwaldring 61, 70550 Stuttgart \\ Germany
}

A numerical model concept for simulating multiphase flow processes is presented. The conceptual model involves the approximation of heterogeneous porous and fractured porous media by application of one-, two- and threedimensional elements in space that can be combined arbitrarily in a single model. The mathematical formulation of multiphase flow is valid for both saturated and unsaturated regions, so that dynamic front propagation behaviour as well as different geology-related retention characteristics can be accounted for in the model. For every Finite Element model problems arise when capillary pressure effects can be neglected and the governing equations are of hyperbolic type. To overcome these difficulties, the differential equations were formulated in terms of pressure and saturation as primary variables. To provide an accurate representation of the resulting saturation-front velocities and to avoid numerical oscillations a modified Petrov-Galerkin method was employed. To verify the code, the BUCKLEY-LEVERETT problem was solved, and together with an adaptive Finite Element algorithm excellent results were obtained.

\section{INTRODUCTION}

Contamination of ground water resources by hazardous substances has become an issue of increasing interest. Petroleum products and halogenated hydrocarbon solvents, ubiquitous in our industrialized society, are among the most serious threats to ground water systems. These organic compounds (non-aqueous phase liquids (NAPL's)) are characterized by their immiscibility with water and air, and low, but toxicologically significant air- and water-phase solubilities. In situations where water, air and NAPL phases occupy the pore space in the subsurface, complex muliphase flow systems are generated.

Modeling of multiphase flow systems involves a number of distinct steps, proceeding from conceptual to mathematical to numerical models. The first task is to develop a conceptual model of the flow system. Based on empirical observations and accepted scientific principles, the conceptual model sets forth general notions about the physical and geometric makeup of the system and its constituents, the important flow and transport processes, and the nature of expected perturbations and constraints. Subsequently a mathematical model can be developed. 
Perhaps the most critical step in a successful modelling effort is the development of the conceptual model. This must strike a proper balance between the complexity of a subsurface system, that will always be only incompletely known, and the level of detail required to address specific engineering issues. The analyst also needs to come to grips with the broad range of space and time scales that may play a role in system definition and performance.

A major issue in numerical modelling is the credibility of computer programs (codes) and simulation results. This is of special importance in technical areas that involve public acceptance. To develop credibility for model predictions, "code verification ${ }^{n}$ exercises are performed. There one seeks to establish, by comparison with independently (often analytically) derived results, that a numerical simulation code does indeed provide accurate solutions for the problem that it was designed to solve. The much more ambitious and difficult task of process interpretation (validation) aims at demonstrating that a numerical model provides a valid representation of physical reality. Verification and process interpretation can only be performed for specific limited conditions; one very obvious limitation being the space and time scales over which the behaviour of a subsurface flow system can be evaluated.

The design and the accurate modelling of the multiphase flow processes e.g. of a subsurface remediation scheme require accurate data of site specific parameters like properties of the porous medium and the present fluids. Also the interactions of the NAPLs with the matrix and with other phases (e.g. water and air) are represented in the relative permeability-and capillary pressure-saturation functions and are important basic inputs although difficult to establish.

The most promising aspects to overcome these problems are offered by well controlled laboratory experiments at different sizes from column to technical scales such as e.g. the VEGASprogram (Research Facility for Subsurface Remediation) (Fig. 1) KoBUS ET. AL. (1993) [8].

\section{GOVERNING EQUATIONS}

Multiphase flow in porous or fractured-porous media is described by a set of nonlinear partial differential equations of two basic categories: the material balance or continuity equation (conservation law) and the equation of motion (Darcy's law). For multiphase fluid flow, these equations must hold for each phase. The conservation law for each phase $\alpha$ is given by a hyperbolic equation which relates porosity, velocity, saturation, density and mass flux. Darcy's law for each phase $\alpha$ is given by an elliptic equation which relates the physical properties of the fluids and pore space with the driving forces of pressure differences and changes in elevation. The multiphase pressure differential equation, including the continuity equation combined with Darcy's law, is widely applied for formulating hydrological problems [3]. 


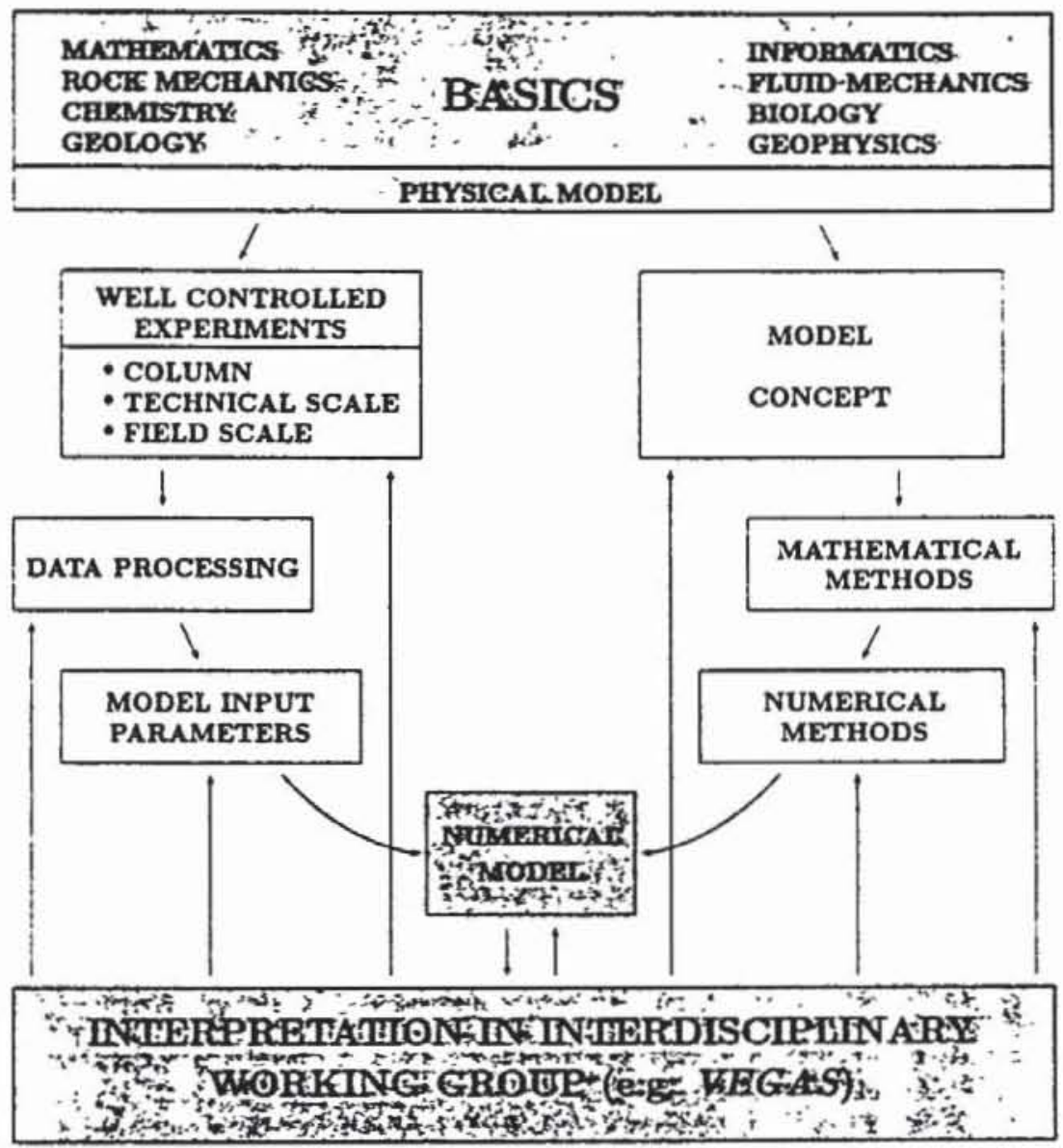

Figure 1: Interdisciplinary cooperation of physical and numerical modelers

$$
\begin{aligned}
& L_{1}\left(p_{\alpha}\right):= \\
& \quad n \varrho_{\alpha} \frac{\partial S_{\alpha}}{\partial t}+S_{\alpha} n \frac{d \varrho_{\alpha}}{d p_{\alpha}} \frac{\partial p_{\alpha}}{\partial t}-\operatorname{div}\left\{k_{r \alpha} \frac{\varrho_{\alpha}}{\mu_{\alpha}} K \cdot\left(\operatorname{grad} p_{\alpha}-\varrho_{\alpha} g\right)\right\}-\varrho_{\alpha} q_{\alpha}=0
\end{aligned}
$$

where $n$ denotes the porosity, $K$ is the permeability tensor, $\varrho_{\alpha}, \mu_{\alpha}, p_{\alpha}, S_{\alpha}$ and $q_{\alpha}$ denote density, viscosity, pressure, saturation and volumetric production rate of phase $\alpha$ respectively. When several fluid phases are present simultaneously in the void space of a porous medium or in a fracture, the presence of any one of these phases will interfere with the flow of all the others. Quantitatively, this mobility reduction is expressed in terms of the relative permeability factor $k_{r \alpha}$. To solve for the unknowns $p_{\alpha}$ and $S_{\alpha}$, more equations are needed. One is given by the fact that the sum of the volume fractions at any point must add to unity:

$$
\sum_{\alpha=1}^{n_{\text {phas }}} S_{\alpha}=1
$$


and the other is given as a relationship for capillary pressures as a function of saturation:

$$
p_{c \alpha \psi}\left(S_{\alpha}\right)=p_{\psi}-p_{\alpha} \quad \psi=1,2, \ldots, n_{\text {phas }} ; \psi \neq \alpha .
$$

The system is nonlinear because $k_{r \alpha}$ and $p_{c}$ are functions of the saturation $S_{\alpha}$. The shapes of these two - often nonlinear - functions are important in determining the nature of the system of equations. This system has a mixed nature and may even change type (elliptic - parabolic - hyperbolic) in certain regions.

From the empirically determined relationship between capillary pressure and saturation, it is observed that the capillary pressure decreases in a strictly monotonic manner with decreasing saturation. An inverse function of the form $S_{\alpha}=S_{\alpha}\left(p_{c \alpha \psi}\right)$ therefore exists. A precondition for the solution of the system of equations is that the capillary pressure $p_{c a \psi} \neq 0$. In the case of fractures (fault zones), the capillary pressure $p_{c \alpha \psi}$ is very small or even equal to zero. The above formulation is therefore not suitable as a mathematical basis for investigating highly heterogeneous media.

A pressure/saturation formulation involving the unknowns $p_{1}, S_{2}, S_{3}, \ldots, S_{n_{\text {phas }}}$, which is valid for an arbitrary capillary pressure, is presented as follows:

$$
\operatorname{grad} p_{\alpha}=\operatorname{grad}\left(p_{1}+\sum_{\Psi=2}^{n_{\text {phas }}} p_{c 1 \alpha}\right)=\operatorname{grad} p_{1}+\sum_{\Psi=2}^{n_{\text {phas }}} \frac{d p_{\mathrm{cl} \alpha}}{d S_{\Psi}} \operatorname{grad} S_{\Psi}
$$

and

$$
\frac{\partial S_{1}}{\partial t}=\frac{\partial}{\partial t}\left(1-\sum_{\Psi=2}^{n_{\text {phas }}} S_{\Psi}\right)=-\sum_{\Psi=2}^{n_{\text {phas }}} \frac{\partial S_{\Psi}}{\partial t}
$$

where the phase $\alpha=1$ represents the fluid phase with the highest affinity to the matrix.

This yields the following expression for phase $\alpha=1$ :

$$
\begin{aligned}
L_{1}\left(p_{1}, S_{\Psi}\right):= & -\operatorname{div}\left\{k_{r 1} \frac{\varrho_{1}}{\mu_{1}} K \cdot \operatorname{grad} p_{1}\right\}+\operatorname{div}\left\{\varrho_{1}^{2} \frac{k_{r 1}}{\mu_{1}} K \cdot g\right\} \\
& +n\left(1-\sum_{\Psi=2}^{n_{\text {phas }}} S_{\Psi}\right) \frac{d \varrho_{1}}{d p_{1}} \frac{\partial p_{1}}{\partial t}-n \varrho_{1} \sum_{\Psi=2}^{n_{\text {phas }}} \frac{\partial S_{\Psi}}{\partial t}-\varrho_{1} q_{1}=0
\end{aligned}
$$

and for the remaining phases $\alpha \geq 2$ :

$$
L_{\alpha}\left(p_{1}, S_{\Psi}\right):=-\operatorname{div}\left\{k_{r \alpha} \frac{\varrho_{\alpha}}{\mu_{\alpha}} K \cdot \operatorname{grad} p_{1}\right\}+\operatorname{div}\left\{\varrho_{\alpha}^{2} \frac{k_{r \alpha}}{\mu_{\alpha}} K \cdot g\right\}
$$




$$
\begin{aligned}
& +n S_{\alpha} \frac{d \varrho_{\alpha}}{d p_{\alpha}} \frac{\partial p_{1}}{\partial t}+n S_{\alpha} \frac{d \varrho_{\alpha}}{d p_{\alpha}} \sum_{\Psi=2}^{n_{\text {phas }}} \frac{d p_{c 1 \alpha}}{d S_{\Psi}} \frac{\partial S_{\Psi}}{\partial t}+n \varrho_{\alpha} \frac{\partial S_{\alpha}}{\partial t} \\
& -\operatorname{div}\left\{k_{r \alpha} \frac{\varrho_{\alpha}}{\mu_{\alpha}} K \cdot \sum_{\Psi=2}^{n_{\text {phas }}} \frac{d p_{c 1 \alpha}}{d S_{\Psi}} \operatorname{grad} S_{\Psi}\right\}-\varrho_{\alpha} q_{\alpha}=0 .
\end{aligned}
$$

Equations 4 and 5 represent a coupled dynamic system of differential equations which describes the simultaneous transport of two or more immiscible fluids in an unsaturated or saturated porous medium. Due to the nonlinear dependence of the capillary pressure and the relative permeability on the degree of saturation, the system of equations behaves in a highly nonlinear manner. This is further intensified by the fact that the physical parameters (such as relative permeability and degree of saturation) as well as the application of the law of motion in the matrix exhibit large differences between various parts such as fracture fault zones or systems with e.g. sand lenses.

\section{NUMERICAL REALIZATION}

The system of equations presented is solved using the Finite Element Method [2]. In discretizing the transient problem, it is appropriate to formulate the time discretization on the basis of an iterative concept. An implicit two-point algorithm developed for this purpose is incorporated into the Newton-type iterative concept for the consistently linearized multiphase problem. The time integration algorithm presented here employs a central difference scheme with a weighting factor of $\theta=0,5$, in which the unknowns $p_{1}$ and $S_{\Psi}$ are solved for a time step of $\Delta t$ (CRANK-NICHOLSON method). With $\theta=1$, the implicit EuLER method (backward difference method) is obtained. In order to describe the multiphase problem in fractured porous media, shape functions are employed within the framework of a standard isoparametric concept for approximating the coordinate field $(X)$, the pressure field of the first phase $\left(p_{1}\right)$, and the saturation field $\left(S_{\Psi}\right)$ of phase $\Psi$. The degrees of freedom of the shape functions are identified by means of discrete nodal coordinates, nodal pressures and nodal saturations.

In order to describe various complicated geological structures (e.g. flow channels, fractures and rock matrix), it is necessary to employ arbitrary combinations of finite elements of different dimensions. Moreover, the finite elements must allow a correct description of the problem defined by the multiphase flow processes.

The modelling system consists of one-, two- and three-dimensional elements with (multi-) linear shapefunctions applied to line elements, plane isoparametric quadrilateral elements and isoparametric hexahedral elements.

As the solutions are computed for each element in turn, the geological parameters may vary from one element to the next. The afore-mentioned combinations of different element types may be applied in a single model. The spatial orientation of the elements is arbitrary in order to simulate complex geological structures [7]. 


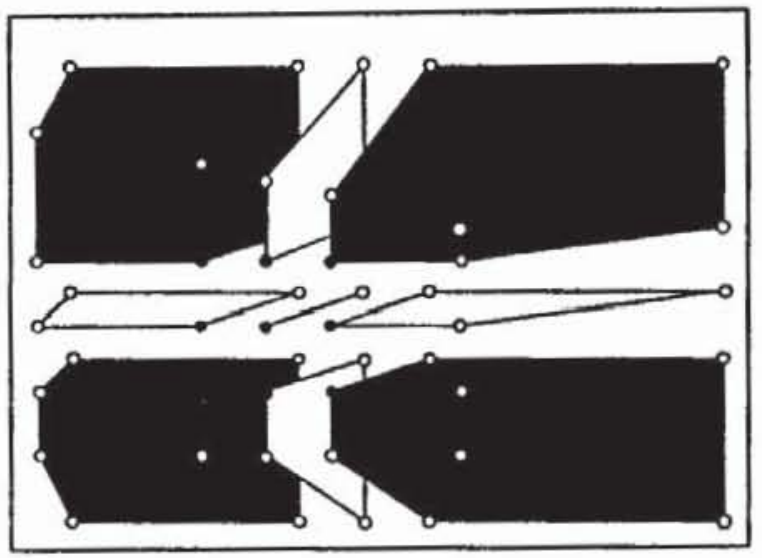

Figure 2: Arbitrary coupling of 1-D, 2-D and 3-D elements

Formally speaking, the coupled, highly nonlinear multiphase equations (Eqns. (4) and (5)) are of the parabolic type when the capillary pressure gradient is significant. If however this gradient becomes very small in relation to the size of the system to be solved, the equations become hyperbolic [4]. The numerical solution obtained from the finite difference and the STANDARD GALERKIN finite element formulation (BUBNOV-GALERKIN method) leads to invalid solutions, particularly when saturation distributions are computed for small capillary pressure effects. These solutions are characterized by physically unjustifiable oscillations of phase shifts as well as an incorrect simulation of front velocities.

In order to ensure convergence of the numerical solution, a modified PETROVGALERKIN method [2] was developed in which the test functions are up to two polynomial degrees higher than the base function (shape function). In the following, the upwinding factors for the quadratic and cubic weighting functions are denoted by $\alpha$ and $\beta$, respectively. As a supplement to this, a lumped finite element formulation was also prepared. This results in a mathematical-numerical formulation which is specially tailored so that the transient multiphase algorithm can be improved by modifying the test function in time and space, thereby guaranteeing convergence.

This type of approach was first proposed by Westerink and Shea (1989) [6] for the predominantly advective transport problem.

\section{VERIFICATION}

The standard method for verifying multiphase processes in the absence of capillary pressure effects represents the Buckley and Leverett (1942) [1] problem, which describes the unsteady displacement of oil by water in a one dimensional, horizontal system. The important point is that the absence of a capillary pressure gradient leads to a formulation of shock in the saturation profile. The following investigations were carried out using 1-D elements. The saturation of the wetting phase (water) and 
the pressure of the nonwetting phase (oil) were prescribed as boundary conditions on the left-hand boundary, with assigned values of $S_{w}=0,8$ and $p_{n}=2 \cdot 10^{5}[\mathrm{~Pa}]$, respectively. A mass flow rate of $m_{n}=1,505 \cdot 10^{-3}\left[\frac{\mathrm{kg}}{\mathrm{s}}\right]$ leaving the system was chosen as the boundary condition on the right-hand boundary.

In order to assess the influence of the relative permeability/saturation relationship on the solution behaviour of the method presented, a Brooks and Corey function was applied. The investigations confirm that the presented multiphase algorithm satisfactorily solves the Buckley-Leverett problem. In order to ensure a good approximation of the front propagation behaviour, it is necessary to employ the quadratic upwinding formulation, whereby a significant reduction in the oscillation of the solution may be achieved with the aid of the lumped finite element or the cubic upwinding formulation. Under these conditions, the conservation of mass is also guaranteed.

In order to reproduce a sharp dynamic front for a nearly $100 \%$ displacement $(100 \%$ saturation for both phases), it was furthermore necessary to develop for 1D elements an adaptive Petrov-Galerkin formulation based on the shown formulation.

A comparison of the numerical and analytical solutions (see Welge (1952) [5]) is given in Fig. 3.
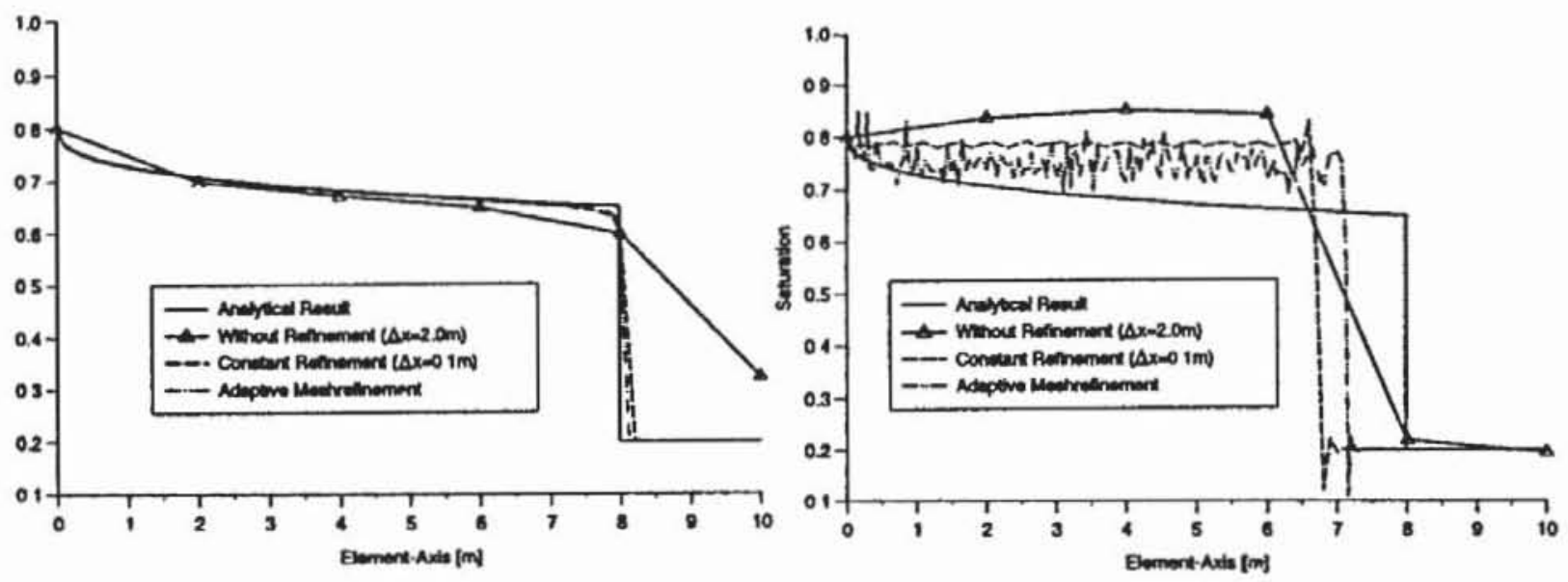

Figure 3: a) Modified Petrov Galerkin Method; b) Standard Galerkin Method

\section{CONCLUSIONS}

A numerical modelling system for multi-phase flow processes in porous and fractured porous media is discribed. To ensure the reproduction of the correct shape of the dynamic saturation front propagation of the phases, a modified Petrov-Galerkin method has been developed.

The numerical model must provide a quantitative description of the relevant processes and mechanisms, based on scientific principles. In cases where sufficient under- 
standing of the important processes has not yet been achieved, as e.g. for multiphase flow in a heterogeneous nature of the subsurface, numerical modelling must be guided by investigations of those processes in laboratory or field experiments.

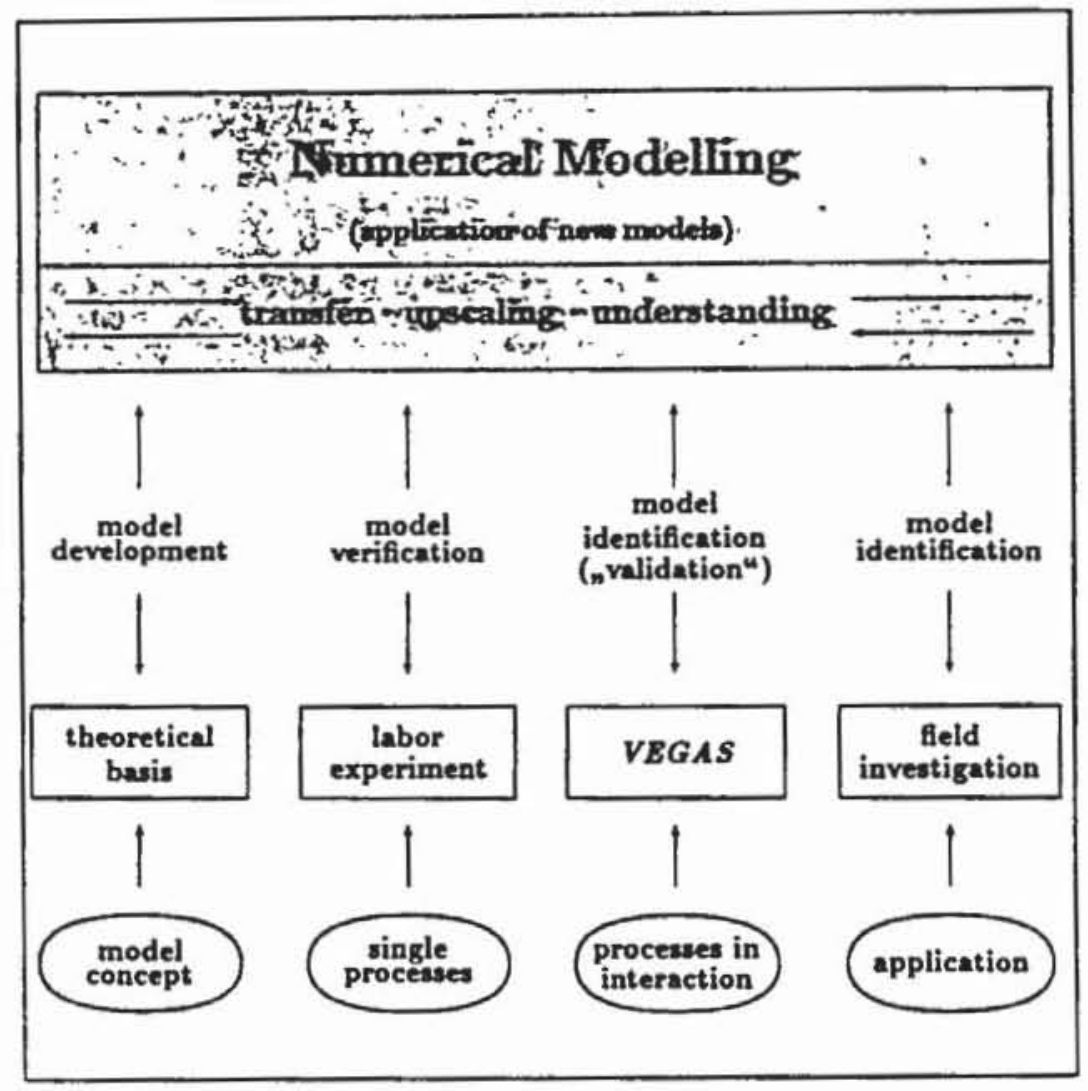

Figure 4: Interpretation of Subsurface Processes by Numerical Models

Presently no method is known or is likely to emerge in the near future, which could demonstrate the accuracy or validity of a numerical model in general "final" terms. This limitation calls for a cautious approach in model applications. Numerical modelling should be open to iterative refinement; models need to be complemented with engineering judgement, and provisions for monitoring and confirmation of system performance must be made. The test facility VEGAS will provide new possibilities for extending the experimental data base for checking and validating multiphase flow and transport models.

\section{ACKNOWLEDGEMENTS}

The first author gratefully acknowledges the financial support of the Deutsche Forschungsgemeinschaft (DFG). A large part of the work reported here was carried out at the Institute of Fluid Mechanics, University of Hannover, FRG and at the LBL, University of California at Berkeley, USA. 


\section{References}

[1] Buckley S.E. and M.C. Leverett: Mechanism of Fluid Displacements in Sands. Trans. AIME, 146:107-116, 1942.

[2] Helmig R. Theorie und Numerik der Mehrphasenströmungen in geklüftetporösen Medien. Bericht Nr.34, Institut für Strömungsmechanik und Elektron.Re chnen im Bauwesen, Universität Hannover, 1993.

[3] Huyakorn P.S. and G.F. Pinder: Computational Methods in Subsurface Flow. Academic Press, London, 1983.

[4] Peaceman D.W.: Fundamentals of Numerical Reservoir Simulation. Elsevier, Amsterdam, 1977.

[5] Welge H.J.: A Simplified Method for Computing Oil Recovery by Gas of Water Drive. Trans. AIME 159, 91-98, 1952.

[6] Westerink J.J. and D. Shea: Consistent Higher Degree Petrov-Galerkin Methods for the Solution of the Transient Convection-Diffusion Equation. International Journal of Num. Methods in Engineering, 28:1077-1101, 1989.

[7] Zielke W. und R. Helmig: Grundwasserströmung und Schadstofftransport FE-Methoden für klüftiges Gestein. In Univ. Fredericiana Karlsruhe (TH), Editor, Wissenschaftliche Tagung "Finite Elemente - Anwendung in der Baupraxis". Verlag Ernst u. Sohn, Berlin, 1991.

[8] Kobus H., Cirpka, O., Barczewski, B., und Koschitzky, H.-P.: Versuchseinrichtung zur Grundwasser- und Altlastensanierung VEGAS - Konzept und Programmrahmen, Mitteilungsheft Nr.82, Institut für Wasserbau, Germany. 